\title{
Sub-acute toxicity and effect of Hwangryunhaedok-tang on human drug-metabolizing enzymes
}

\author{
Seong Eun Jin ${ }^{1}$, Mee-Young Lee ${ }^{1}$, Chang-Seob Seo ${ }^{1}$, Hyeun-Kyoo Shin ${ }^{1}$, Jae-Woo \\ $\mathrm{Cho}^{2}$, Hyekyung $\mathrm{Ha}^{1, *}$ \\ ${ }^{1} \mathrm{~K}$-herb Research Center, Korea Institute of Oriental Medicine \\ ${ }^{2}$ Pathology Research Center, Korea Institute of Toxicology
}

\begin{abstract}
Objectives: Hwangryunhaedok-tang (HHT; Huanglianjiedu-tang, Orengedoku-to), a traditional herbal formula, is used for treating inflammation, hypertension, gastritis, liver dysfunction, cerebrovascular diseases, dermatitis and dementia. The objective of this study was to assess the sub-acute toxicity of HHT in Sprague-Dawley (SD) rats, and its effect on the activities of human microsomal cytochrome P450s (CYP450s) and UDP-glucuronosyltransferases (UGTs). Methods: Male and female SD rats were orally administered HHT once daily at doses of 0, 500, 1000 and 2000 $\mathrm{mg} / \mathrm{kg}$ for 4 weeks. We analyzed mortality, clinical observations, body weight, food consumption, organ weights, urinalysis, hematology, serum biochemistry, and histopathology. The activities of major human CYP450s (CYP1A2, CYP3A4, CYP2B6, CYP2C9, CYP2C19, CYP2D6, and CYP2E1) and UGTs (UGT1A1, UGT1A4, and UGT2B7) were assessed using in vitro fluorescence- and luminescence-based enzyme assays, respectively.

Results: No toxicologically significant changes related to the repeated administration of HHT were observed in both male and female SD rats. The no observed adverse effect level (NOAEL) value was more than $2000 \mathrm{mg} / \mathrm{kg} / \mathrm{day}$ for both sexes. HHT inhibited the activities of human microsomal CYP1A2, CYP2C19, CYP2D6, and CYP2E1, whereas it weakly inhibited the activities of CYP2B6, CYP2C9, CYP3A4, and UGT1A1. In addition, HHT negligibly inhibited the activities of human microsomal UGT1A4 and UGT2B7 with $\mathrm{IC}_{50}$ values in excess of $1000 \mu \mathrm{g} / \mathrm{mL}$.

Conclusions: Our findings indicate that HHT may be safe for repeated administration up to 4 weeks. In addition, these findings provide information on the safety and effectiveness of HHT when co-administered with conventional drugs.
\end{abstract}

Key Words : Hwangryunhaedok-tang, sub-acute toxicity, cytochrome P450, UDP-glucuronosyltransferase, herb-drug interactions

\section{Introduction}

Herbal medicines have been used for the treatment of a multitude of diseases, and are often used in conjunction with conventional drugs. Some herbal medicines have shown significant side effects even though they comprise natural products. However, there is limited scientific evidence to validate the safety of majority of the herbal medicines ${ }^{1)}$. In particular, the co-administration of herbal formulas with conventional drugs raises even deeper safety concerns because herbal formulas comprise various herbal medicines and constituents.

Herbal medicines and their constituents can alter the activity or expression of drug-metabolizing enzymes, which can lead to changes in the efficacy

\footnotetext{
- Received : 6 April $2017 \quad \cdot$ Revised : 29 May 2017 Accepted : 29 May 2017

- Correspondence to : Hyekyung Ha

K-herb Research Center, Korea Institute of Oriental Medicine

1672 Yuseong-daero, Yuseong-gu, Daejeon 34054, Republic of Korea

Tel : +82-42-868-9513, Fax : +82-42-864-2120, E-mail : hkha@kiom.re.kr
} 
and toxicity of conventional drugs. Therefore, information on the potential of herbal medicines to inhibit or induce the activity or expression of drug-metabolizing enzymes when co-administered with conventional drugs is important.

In general, studies on the safety of herbal medicines, including herbal formulas, should include at least in vitro and in vivo genotoxicity assays, long-term rodent carcinogenicity tests, reproductive and developmental toxicity studies, and investigation of their effects on drug-metabolizing enzymes ${ }^{2}$.

Drug metabolism is divided into phase I and phase II transformation reactions. Cytochrome P450 (CYP450), a class of phase I enzymes, exists as a superfamily of heme containing enzymes that catalyze the oxidative metabolism of various endogenous and xenobiotic substrates ${ }^{3)}$. Approximately $73 \%$ of all known drugs are metabolized by hepatic CYP450 $0^{4}$. In particular, CYP1A2, CYP2C9, CYP2C19, CYP2D6, and CYP3A4 are responsible for approximately $80 \%$ of the known oxidative drug reactions, and they are highly subjected to inhibition owing to their broad specificity for structurally diverse substrates ${ }^{4}$. UDP-glucuronosyltransferases (UGT), a class of phase II enzymes, catalyze the conjugation of glucuronic acid to exogenous substances and endogenous components ${ }^{5)}$. The human UGTs are generally categorized into UGT1A, UGT2A, and UGT2B subfamilies ${ }^{6}$.

Hwangryunhaedok-tang (HHT; Huanglianjiedu-tang, Orengedoku-to) is a traditional herbal formula comprising Coptidis Rhizoma, Scutellariae Radix, Phellodendri Cortex, and Gardeniae Fructus ${ }^{7}$. HHT has been used for treating inflammation, hypertension, gastritis, liver dysfunction, cerebrovascular diseases, dermatitis, and dementia ${ }^{8,9)}$. In our previously study, the sub-chronic toxicity of HHT in Sprague-Dawley (SD) rats was reported. Under our experimental conditions, the no observed adverse effect level (NOAEL) of HHT for rats of either sex was 750 $\mathrm{mg} / \mathrm{kg} / \mathrm{day}^{10}{ }^{10}$. However, other studies on the toxic and influence of HHT on drug-metabolizing enzymes are required to evaluate the safety of HHT.

In this study, we evaluated the sub-acute toxicity of HHT in SD rats, and the effects of HHT on the activities of major human CYP450s (CYP1A2, CYP2B6, CYP2C9, CYP2C19, CYP2D6, CYP2E1, and CYP3A4) and UGTs (UGT1A1, UGT1A4, and UGT2B7) drug metabolizing enzymes.

\section{Materials and methods}

\section{Chemicals and materials}

Vivid ${ }^{\circledR}$ CYP450 Screening Kits $\left(\right.$ Vivid $^{\circledR}$ CYP1A2 Blue, Vivid $^{\circledR}$ CYP2B6 Blue, Vivid ${ }^{\circledR}$ CYP2C9 Blue, Vivid $^{\circledR}$ CYP2C19 Blue, Vivid ${ }^{\circledR}$ CYP2D6 Blue, Vivid $^{\circledR}$ CYP2E1 Blue, and Vivid ${ }^{\circledR}$ CYP3A4 Green) were purchased from Invitrogen Co. (Camarillo, CA, USA). These kits use 7-ethoxy-methyloxy-3 -cyanocoumarin (EOMCC) as a substrate for CYP1A2, CYP2D6, CYP2C19, and CYP2E1. Additionally, di(benzyloxymethoxy)fluorescein (DBOMF) was used as a substrate for CYP3A4, and 7 -benzyloxy-4-trifluoromethylcoumarin (BOMCC) was used as a substrate for CYP2B6 and CYP2C9. UGT-Glo $^{\text {TM }}$ UGT1A1 and UGT2B7 Screening Systems were purchased from Promega (Madison, WI, USA). The recombinant human UGT1A4 enzyme was purchased from Corning, Inc. Life Sciences (Tewksbury, MA, USA). a-Naphthoflavone, ketoconazole, miconazole, sulfaphenazole, quinidine, sodium diethyldithiocarbamate trihydrate, diclofenac, and lopinavir were obtained from Sigma Chemical Co. (St. Louis, MO, USA). All other chemicals were of analytical grade.

\section{Preparation of $\mathrm{HHT}$}

Preparation of HHT was performed as previously reported $^{11)}$. In brief, we prepared the HHT extract in our laboratory. The four medicinal herbs that constitute HHT, Coptis chinensis (Coptidis Rhizoma, 
China), Scutellaria baicalensis (Scutellariae Radix, Jeongseon, Korea), Phellodendron chinensis (Phellodendri Cortex, China), and Gardenia jasminoides (Gardeniae Fructus, Muju, Korea) were purchased from Omniherb (Yeongcheon, Korea) and HMAX (Jecheon, Korea). A mixture of the chopped medicinal herbs was extracted in distilled water (10 times the sample amount) at $100^{\circ} \mathrm{C}$ for $2 \mathrm{~h}$ under pressure (98 $\mathrm{kPa}$ ). The solution was evaporated to dryness and freeze-dried. This sample was named as 'Voucher specimens (2008-KE-20-1 KE-20 4)', and these specimens have been deposited at the K-herb Research Center of Korea Institute of Oriental Medicine. The high performance liquid chromatography (HPLC) profile of HHT has been previously reported $^{11)}$. In brief, the contents HHT extract are as follows: geniposide $36.54 \pm 0.27 \mathrm{mg} / \mathrm{g}$, baicalein $30.24 \pm 0.72 \mathrm{mg} / \mathrm{g}$, palmatine $10.34 \pm 0.47 \mathrm{mg} / \mathrm{g}$, berberine $1.35 \pm 0.02 \mathrm{mg} / \mathrm{g}$ and coptisine $0.97 \pm$ $0.02 \mathrm{mg} / \mathrm{g}$.

\section{Animals}

Each of 20 male and female specific pathogen -free SD rats (six-week-old) were obtained from Orient Bio Inc. (Seongnam, Republic of Korea). The rats underwent one week of acclimatization prior to study initiation. Five animals were used per group. Two or three animals were housed per polycarbonate cage stainless-steel wire-mesh cage, and they were allowed ad libitum access to sterilized tap water and commercial rodent chow (Purina Co., Pyeongtaek, Republic of Korea). The animals were maintained in environmentally-controlled rooms at $23 \pm 3^{\circ} \mathrm{C}$ under relative humidity of $50 \pm 10 \%$, with a $12 \mathrm{~h}$ light-dark cycle (artificial lighting from 08:00 to 20:00), and 10-20 air changes per hour. This study was conducted at the Korea Testing and Research Institute, and the protocol was approved by the Institutional Animal Care and Use Committee according to the "Guidelines for Toxicity Tests of
Drugs and Related Materials, Document \#2009-116" prepared by Korea Food and Drug Administration (2009). The approval number is \#G11025.

\section{Dose selection and animal treatment}

In our previous single-dose study of HHT, no treatment-related toxic changes were observed at the highest dose $(2000 \mathrm{mg} / \mathrm{kg} /$ day $)$. Therefore, 0, 500, 1000 , and $2000 \mathrm{mg} / \mathrm{kg} /$ day of HHT were selected for this 4-week repeated dose study. Healthy male and female rats were randomly assigned to four experimental groups. Each group consisted of five rats of each sex. HHT was suspended in distilled water, and freshly prepared daily before treatment. The daily application volume $(5 \mathrm{~mL} / \mathrm{kg})$ of HHT was calculated in advance based on the most recently recorded body weights of individual animals. The rats were orally administered HHT for 4 weeks, while distilled water was given to the animals in the vehicle control group.

\section{Clinical observations}

During the experimental period, the rats were observed twice daily for clinical signs and mortality. All clinical signs were recorded individually for type, observation day/time, and duration using the Path/Tox System 4.2.2 (Xybion Medical Systems Corp., USA). Body weight and food consumption were recorded weekly.

\section{Urinalysis and hematology}

During the last week of the experiment, urinalysis was conducted to assess the urine volume, specific gravity (SG), $\mathrm{pH}$, and urobilinogen (URO) using Multistix 10 SG (Bayer, NJ, USA) and urine chemical analyzer (Clinitek-500, GMI, MN, USA).

On day 28 , the rats were fasted overnight, and they were anesthetized with isoflurane and sacrificed. Blood samples were drawn from the posterior vena cava and collected in complete blood 
count (CBC) bottles containing EDTA-2K (Sewon Medical Co., Republic of Korea). The samples were analyzed using an ADVIA120 Hematology System (Bayer) to determine the white blood cell count (WBC), red blood cell count (RBC), hemoglobin concentration (HGC), hematocrit (HCT), mean corpuscular volume (MCV), mean corpuscular hemoglobin $(\mathrm{MCH})$, mean corpuscular hemoglobin concentration (MCHC), platelet (PLT), reticulocyte (RET), neutrophils, lymphocytes, monocytes, basophils, and large unstained cells (LUC). Prothrombin time (PT) and activated partial thromboplastin time (APTT) were determined in blood samples treated with $3.2 \%$ sodium citrate using a coagulometer (ACL 9000, Instrumentation Laboratory Spa, Milan, Italy).

\section{Serum biochemistry}

The blood samples were centrifuged at 3,000g for $10 \mathrm{~min}$ and analyzed for glucose (GLU), blood urea nitrogen (BUN), creatinine (CREA), total protein (TP), albumin (ALB), albumin/globulin ratio $(\mathrm{A} / \mathrm{G})$, total cholesterol (TCHO), triglyceride (TG), phospholipid (PL), alanine aminotransferase (ALT), aspartate aminotransferase (AST), alkaline phosphatase (ALP), total bilirubin (TBIL), and creatine kinase (CK) using an autoanalyzer (Toshiba 200 FR NEO, Toshiba Co., Japan).

\section{Necropsy}

Complete gross necropsy was performed on all rats. The absolute weights of the brain, pituitary gland, liver, spleen, kidney, lung, heart, thymus, salivary glands, adrenal glands, thyroid/parathyroid, salivary vesicle (male), prostate (male), testes (male), epididymis (male), ovaries (female), and uterus/cervix (female) were measured, and the relative organ weights (organ-to-body weight ratios) were calculated.

\section{Histopathology}

The liver, kidneys, mandibular lymph nodes, and uterus/cervix samples were fixed in $10 \%$ neutral -buffered formalin, sectioned at $4 \mu \mathrm{m}$, and stained with hematoxylin and eosin (H\&E, Sigma). The tissues were subsequently mounted and coverslipped using the Dako mounting medium (Invitrogen, USA).

\section{Cytochrome P450 isozyme assay}

The assays were performed using Vivid $^{\circledR}$ CYP450 Screening Kits according to the protocol provided by the manufacturer and previously described methods ${ }^{12)}$. Vivid $^{\circledR}$ CYP450 Screening Kits are designed to assess the metabolic activity of the predominant human CYP450s (CYP1A2, CYP3A4, CYP2B6, CYP2C9, CYP2C19, CYP2D6, or CYP2E1) involved in hepatic drug metabolism. A test sample of $40 \mu \mathrm{L}$ diluted in solvent, a positive inhibition control or a solvent control was added to each well. The solutions were mixed after adding $50 \mu \mathrm{L}$ Master Pre-Mix containing P450 BACULOSOMES ${ }^{\circledR}$ in the Vivid $^{\circledR}$ CYP450 Reaction Buffer and Regeneration System (consisting of glucose-6-phosphate and glucose-6-phosphate dehydrogenase), and the plate was incubated for 20 min to allow the samples to interact with the CYP enzymes. The Regeneration System converts $\mathrm{NADP}^{+}$into NADPH. After pre-incubation, the reaction was initiated by adding $10 \mu \mathrm{L}$ of the mixture of Vivid $^{\circledR}$ Substrate and $\mathrm{NADP}^{+}$. For CYP3A4, the fluorescence intensity was measured using an EnVision 2103 Multilabel Reader (PerkinElmer Inc., MA, USA) for $15 \mathrm{~min}$ at excitation and emission wavelengths of 485 and 535 nm, respectively. For CYP1A2, CYP2B6, CYP2C9, CYP2C19, CYP2D6, or CYP2E1, the fluorescence intensity was measured for $60 \mathrm{~min}$ at excitation and emission wavelengths of 415 and $460 \mathrm{~nm}$, respectively, using a SpectraMax ${ }^{\circledR}$ i3 (Molecular Devices Co., Sunnyvale, CA, USA). 
The inhibition percentage (\%) was obtained by the following equation: $\%$ Inhibition $=\left[1-\left(\mathrm{S}_{1}-\right.\right.$ $\left.\left.\mathrm{S}_{0}\right) /\left(\mathrm{C}_{1}-\mathrm{C}_{0}\right)\right] \times 100$, where $\mathrm{C}_{1}$ is the fluorescence of the control after incubation, $\mathrm{C}_{0}$ is the initial fluorescence of the control, $\mathrm{S}_{1}$ is the fluorescence of the test sample after incubation, and $\mathrm{S}_{0}$ is the initial fluorescence of the test sample in the linear section.

The background fluorescence of the herbal formulas was corrected by subtracting the values obtained from the incubation without substrates. CYP450 inhibition of the sample was expressed in terms of $\mathrm{IC}_{50}$, as calculated from the log-dose inhibition curve (SigmaPlot, Ver. 12.5, Systat Software, Inc., CA, USA). The data are expressed as the means \pm standard error of the mean (SEM; $n$ =3). a-Naphthoflavone, ketoconazole, sulfaphenazole, quinidine, and sodium diethyldithiocarbamate trihydrate were used as positive controls for CYP1A2, CYP3A4, CYP2C9, CYP2D6, and CYP2E1, respectively. Miconazole was used as a positive control for CYP2B6 and CYP2C19.

\section{UDP-glucuronosyltransferase isozyme assay}

The assays were performed using the UGT-Glo ${ }^{\mathrm{TM}}$ Screening Systems according to the manufacturer's protocol and previously described methods ${ }^{12)}$. The assay systems provide a luminescent method for measuring the activity of UGTs. Two glucuronidation reactions were set up in parallel to measure UGT activity. Both reactions contained a source of UGTs (UGT1A1, UGT1A4, or UGT2B7) and the proluciferin substrate (UGT Multienzyme Substrate or UGT1A4 Substrate); however, only one of them contained the uridine 5'-diphosphoglucuronic acid (UDPGA) cofactor. Ten microliters of $4 \times$ concentrated test sample, a positive inhibition control, or a solvent control was added to each well. Then, $10 \mu \mathrm{L}$ of UDPGA (Plus-UDPGA reaction set) or distilled water (Minus-UDPGA reaction set) was added to the relevant wells. Twenty microliters of the prepared $2 \times$ control reaction mixture (Minus-UGT enzyme) and the $2 \times$ UGT reaction mixture (UGT1A1, UGT1A4, or UGT2B7) were added to the appropriate wells. The reaction solution was mixed and incubated at $37^{\circ} \mathrm{C}$ for 90,180 , or 60 min, respectively, for UGT1A1, UGT1A4, or UGT2B7. The final contents of the reactant were $0.1 \mathrm{mg} / \mathrm{mL}$ UGT enzyme and $20 \mu \mathrm{M}$ enzyme substrate in the presence or absence of $4 \mathrm{mM}$ UDPGA. After incubation, $40 \mu \mathrm{L}$ of the reconstituted Luciferin Detection Reagent plus D-cysteine was added to all wells. After 20 min of incubation at room temperature, the luminescence signal was detected using a SpectraMax ${ }^{\circledR}$ i3.

The detected data were converted to the calculated difference using the following percentage of substrate consumed (\%SC) equation: \% Substrate consumed $=$ (background corrected difference) $/($ average minus-UDPGA values) $\times 100$. The inhibition percentage (\%) was obtained via the following equation: \% Inhibition $=\left[1-\left(\mathrm{S} / \mathrm{C}_{\mathrm{AVR}}\right)\right] \times 100$, where $\mathrm{S}$ is the $\% \mathrm{SC}$ of each sample or the control wells, and $\mathrm{C}_{\mathrm{AVR}}$ is the average $\% \mathrm{SC}$ of the control wells. The UGT inhibition of each sample was expressed in terms of $\mathrm{IC}_{50}$ as calculated using SigmaPlot (Ver. 12.5), which is capable of generating a four parameter logistic curve fit. The data are expressed as the means $\pm \operatorname{SEM}(n=2)$.

\section{Statistics analysis}

Data collected during the study were examined for homogeneity of variance using Bartlett's test. In case of no significant deviations from homogeneity of variance, one-way ANOVA was conducted at $a=$ 0.05 . In case of significance, multiple comparisons were performed using Dunnett's test to determine which pairs of groups were significantly different. When significant deviations from homogeneity of variance were observed, a non-parametric comparison 
test was conducted using Kruskal-Wallis test. When a significant difference was observed in Kruskal -Wallis test, Dunn's rank sum test was performed to determine the specific pairs. Statistical analyses were conducted using the Path/Tox System (Ver. 4.2.2, Xybion Medical Systems Corp.). The level of significance was taken as $P<0.05$ or 0.01 .

\section{Results}

1. Mortality and clinical signs

A

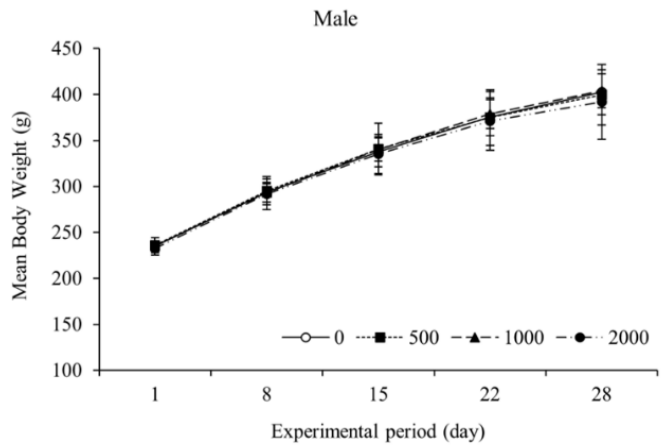

No HHT treatment-related mortality occurred in either sex. Salivation was observed in all male rats in the $2000 \mathrm{mg} / \mathrm{kg} /$ day group, and in one case in the $1000 \mathrm{mg} / \mathrm{kg} /$ day group. In female rats, salivation was observed in two cases in the $2000 \mathrm{mg} / \mathrm{kg} / \mathrm{day}$ group. In addition, loss of fur was detected in some rats.

2. Changes in body weight, food consumption, and organ weight

During the experimental period, no significant

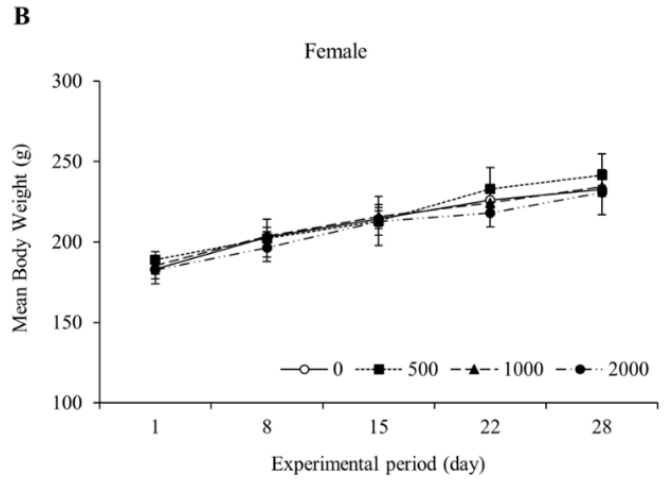

Fig. 1. Body weight changes of rats treated orally with Hwangryunhaedok-tang for 4 weeks.

Body weight changes of male (A) and female (B) rats treated with Hwangryunhaedok-tang at dose levels of 0 (O), 500 ( $\mathbf{\square}), 1000$ ( $\mathbf{\Delta}$ ), and 2000 (-) $\mathrm{mg} / \mathrm{kg} / \mathrm{day}$ for 4 weeks. The values are presented as means $\pm \mathrm{SD}$.

A

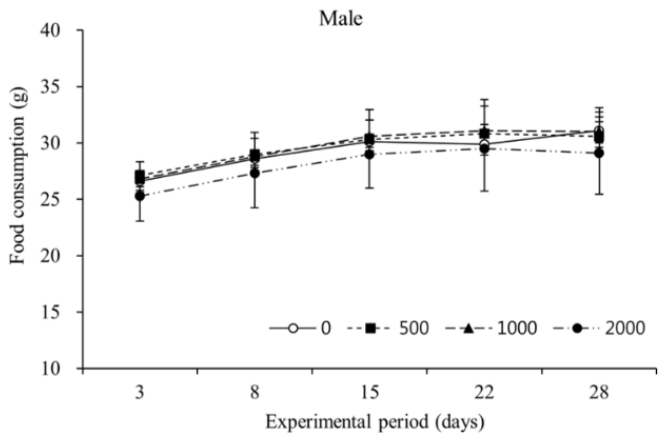

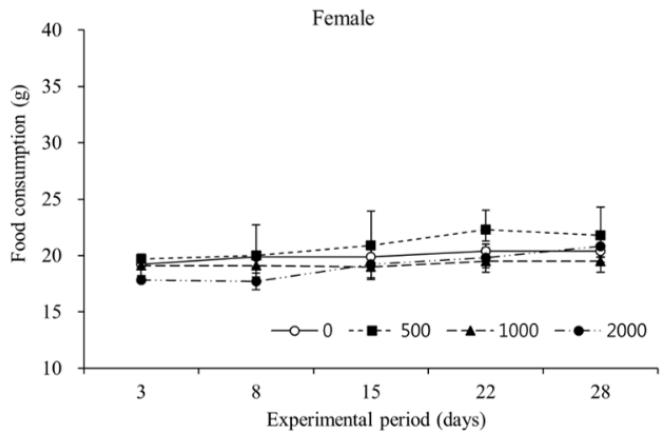

Fig. 2. Food consumption of rats treated orally with Hwangryunhaedok-tang for 4 weeks.

Food consumption of male (A) and female (B) rats treated with Hwangryunhaedok-tang at dose levels of 0 (O), 500 ( $\mathbf{\square}), 1000$ ( $\mathbf{\Delta}$ ), and 2000 $(\bullet) \mathrm{mg} / \mathrm{kg} / \mathrm{day}$ for 4 weeks. The values are presented as means \pm SD. 
differences in body weight and food consumption were observed between the vehicle control group and HHT-treated groups for either sex (Fig. 1 and 2). In male rats, there were no significant differences in relative organ weights between the vehicle control and HHT-treated groups (Table 1). In contrast, the relative spleen weight was significantly increased in the 1000 and $2000 \mathrm{mg} / \mathrm{kg} /$ day female groups when compared with that in the vehicle control group (Table 1). Additionally, the relative kidney weights were significantly increased in the HHT-treated female groups, and relative ovaries weights were significantly decreased in the 1000 and 2000 $\mathrm{mg} / \mathrm{kg} /$ day female groups when compared with those in the vehicle control group (Table 1).

\section{Urinalysis and hematology}

In the $1000 \mathrm{mg} / \mathrm{kg} /$ day female group, volume of urine was increased when compared with those in the vehicle control group, but was within the normal range. There were no significant changes found in both male and female rats among any of the HHT-treated groups for volume, SG, $\mathrm{pH}$ and URO when compared with the vehicle control group (Table 2).

In both male and female rats treated with 2000 $\mathrm{mg} / \mathrm{kg} /$ day of HHT, an increase in bilirubin $(+1 \sim$ +2 ) was detected (male, $n=5$; female, $n=5$ ) when compared with that in the vehicle control group. and green urine were observed (male, $\mathrm{n}=4$; female, $\mathrm{n}=$ 3; data not shown). In the $1000 \mathrm{mg} / \mathrm{kg} /$ day male group, green urine was observed in one case, and the percentage of MCHC was significantly increased when compared with that in the vehicle control group (Table 3). In the $2000 \mathrm{mg} / \mathrm{kg} /$ day female group, significant decrease of $\mathrm{RBC}$ and increase in the percentage of RET were detected when compared with those in the vehicle control group (Table 3).

\section{Serum biochemistry}

No significant differences in serum biochemical values were observed in rats treated with HHT (Table 4).

\section{Necropsy findings}

Dark discoloration of the kidneys was observed at $1000 \mathrm{mg} / \mathrm{kg}$ /day (male, $\mathrm{n}=1$; female, $\mathrm{n}=2$ ) and $2000 \mathrm{mg} / \mathrm{kg} /$ day (male, $\mathrm{n}=5$; female, $\mathrm{n}=5$ ) of HHT in both sexes. In addition, the male and female rats treated with $2000 \mathrm{mg} / \mathrm{kg} /$ day of HHT showed dark discoloration of the liver (male, $\mathrm{n}=4$; female, $\mathrm{n}=5$ ).

\section{Histopathological examination}

HHT treatment did not induce histopathological changes in the liver, kidneys, mandibular lymph nodes, and uterus/cervix at less than 2000 $\mathrm{mg} / \mathrm{kg} /$ day (data not shown).

\section{Effects of HHT on the CYP450s activities}

In vitro fluorescence-based CYP450 assays were carried out to investigate the influence of HHT on the activities of human CYP1A2, CYP2B6, CYP2C9, CYP2C19, CYP2D6, CYP2E1, and CYP3A4. a -Naphthoflavone, sulfaphenazole, quinidine, diethyldithiocarbamate, and ketoconazole inhibited the activities of CYP1A2, CYP2C9, CYP2D6, CYP2E1, and CYP3A4 in a dose-dependent manner, with $\mathrm{IC}_{50}$ values of $396.84 \mathrm{nM}, 241.55 \mathrm{nM}, 3.55$ $\mathrm{nM}, 29.77 \mu \mathrm{M}$, and $1.55 \mathrm{nM}$, respectively (Fig. 3 and Table 5). Miconazole inhibited the activities of CYP2B6 and CYP2C19 in a dose-dependent manner, with $\mathrm{IC}_{50}$ values of $2.95 \mu \mathrm{M}$ and $1.47 \mu \mathrm{M}$, respectively (Fig. 3 and Table 5).

HHT potently inhibited the CYP2D6 activity, with an $\mathrm{IC}_{50}$ value of $0.87 \mu \mathrm{g} / \mathrm{mL}$, followed by the activities of CYP2C19, CYP2E1, and CYP1A2, with $\mathrm{IC}_{50}$ values of $96.61 \mu \mathrm{g} / \mathrm{mL}, 144.94 \mu \mathrm{g} / \mathrm{mL}$, and 

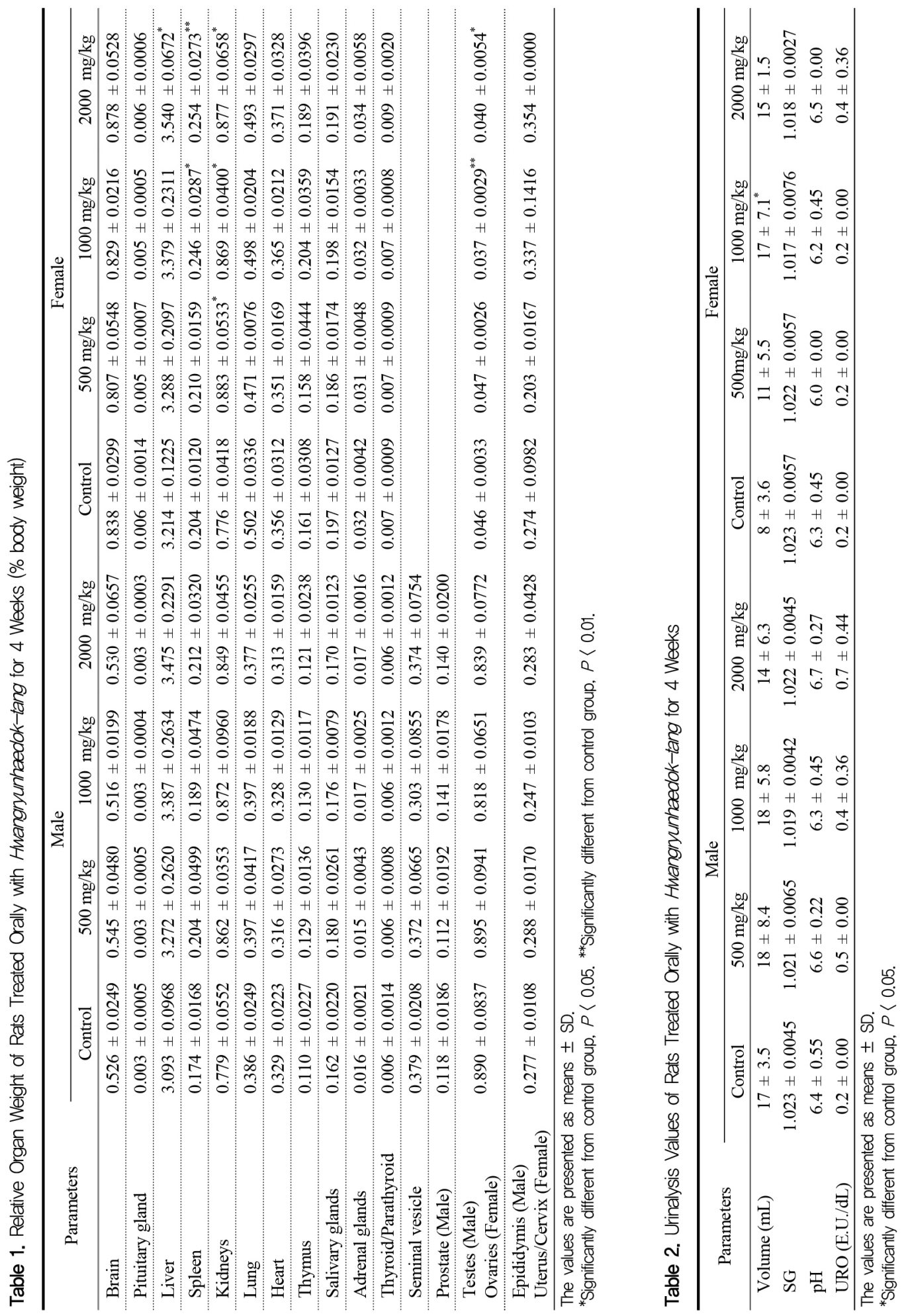


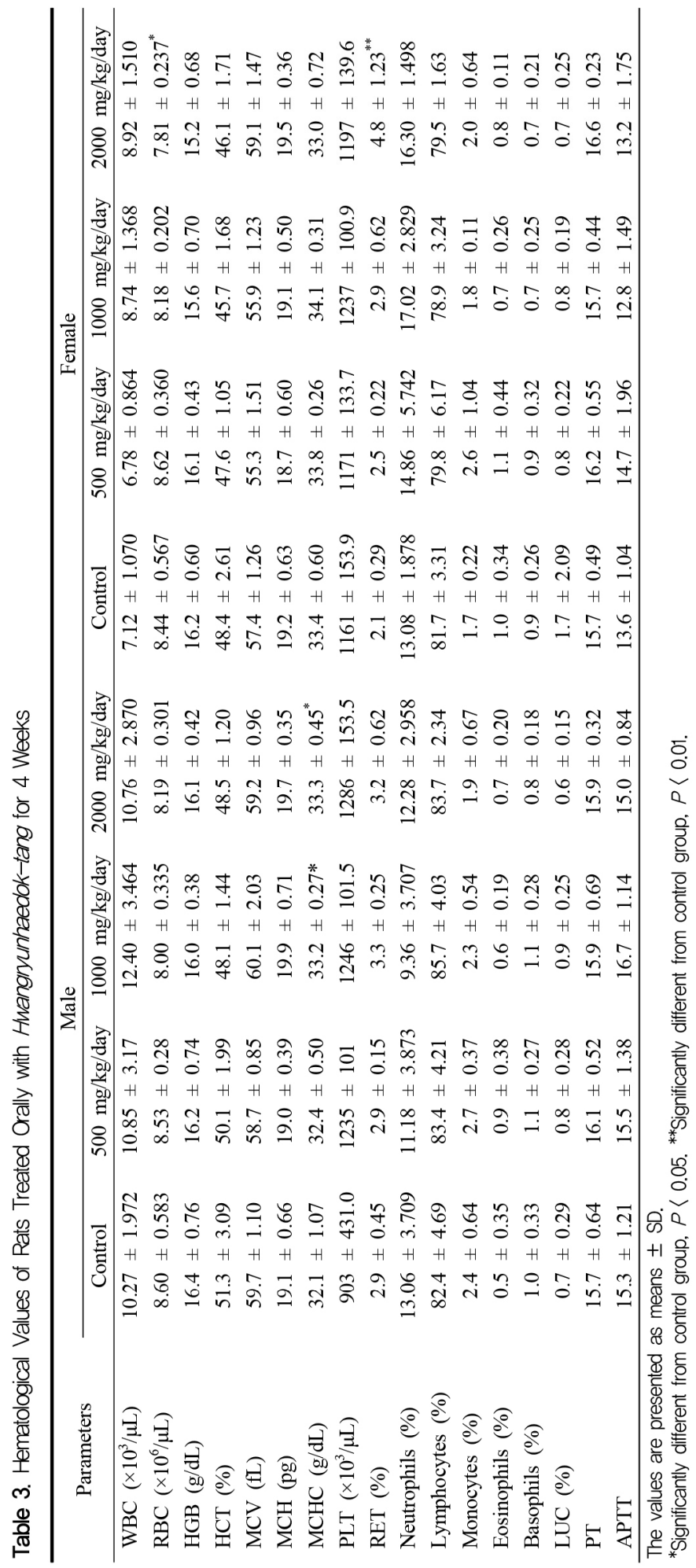



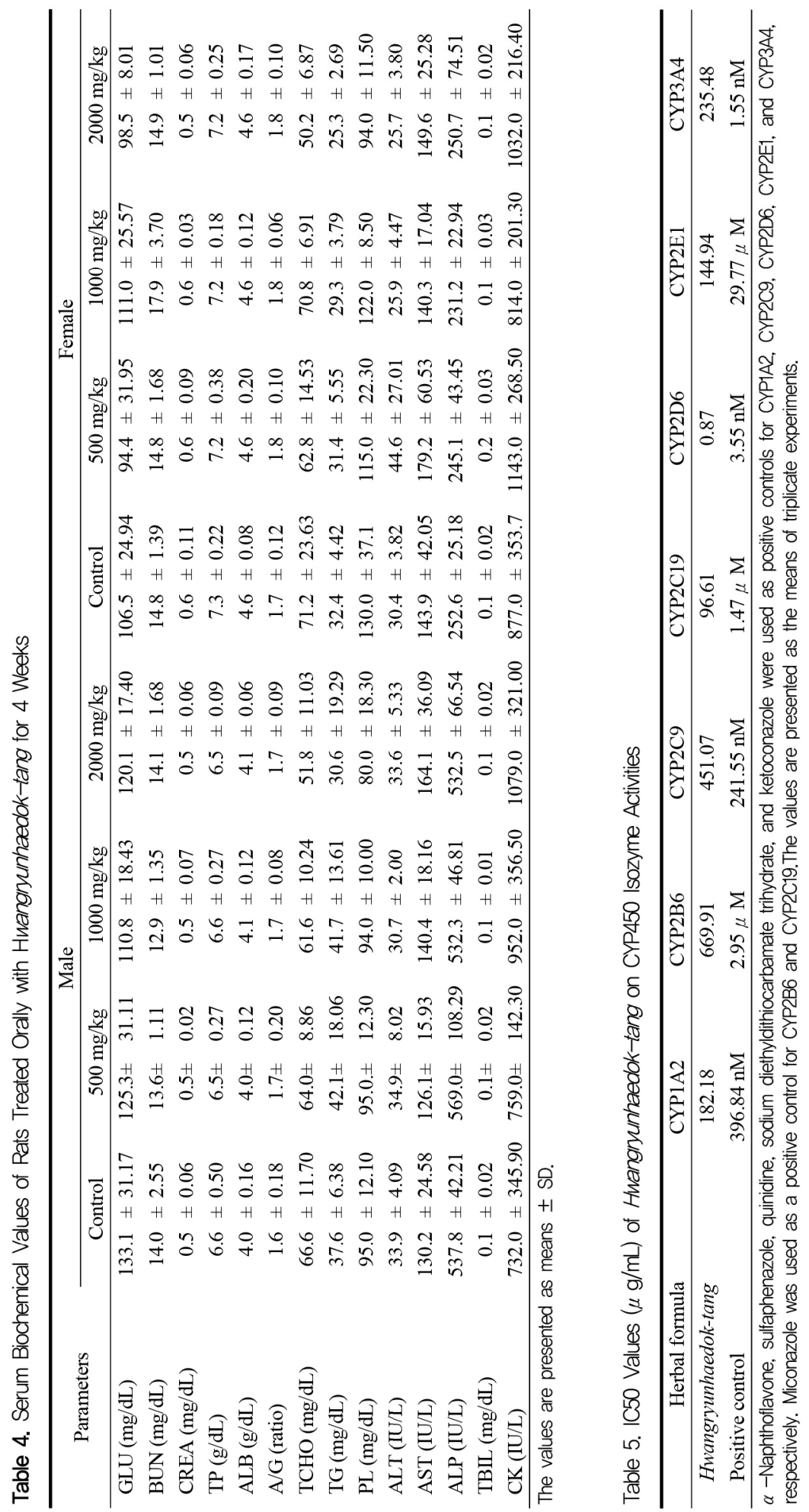
A

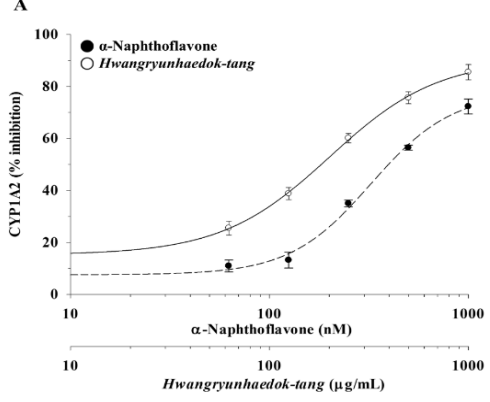

C

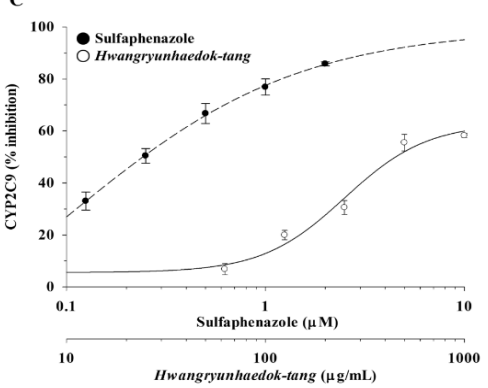

E

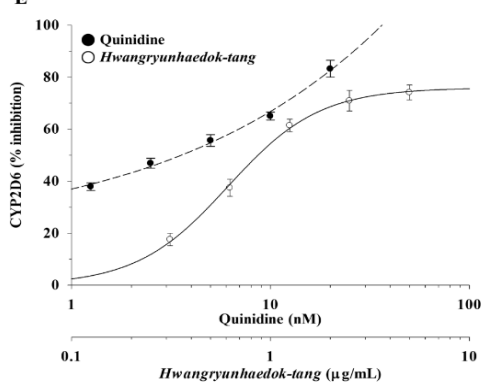

G

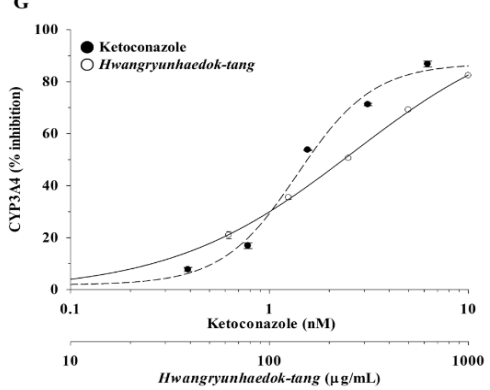

B

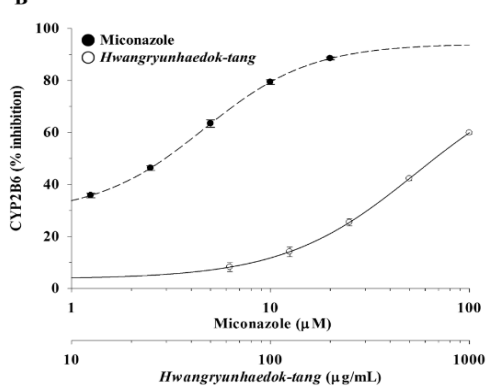

D

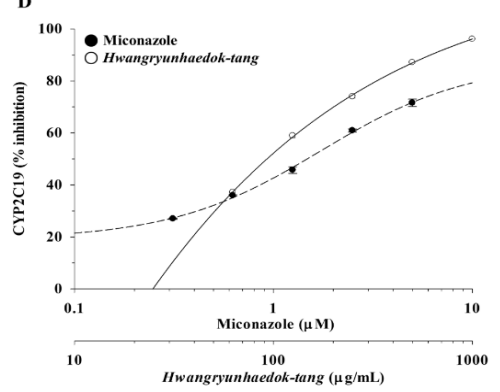

F

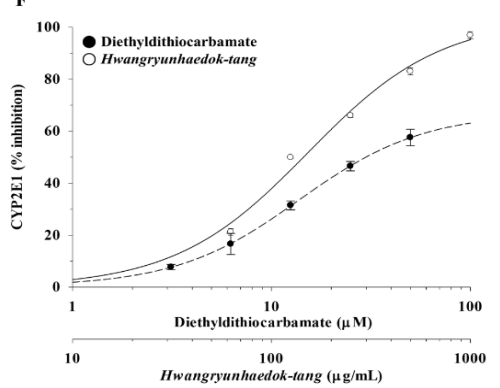

Fig. 3. The effects of Hwangryunhaedok-tang on the activities of CYP1A2 (A), CYP2B6 (B), CYP2C9 (C), CYP2C19 (D), CYP2D6 (E), CYP2E1 (F), and CYP3A4 (G).

Fluorescence-based enzyme assays of human microsomal CYP450 isozymes were established in vitro. $\alpha$-Naphthoflavone, sulfaphenazole, quinidine, sodium diethyldithiocarbamate trihydrate, and ketoconazole were used as positive controls for CYP1A2, CYP2C9, CYP2D6, CYP2E1 and CYP3A4, respectively. Miconazole was used as a positive control for CYP2B6 and CYP2C19. The data are presented as the means \pm SEM ( $n=3$ ). 
$182.18 \mathrm{\mu g} / \mathrm{mL}$, respectively (Fig. 3 and Table 5). However, HHT showed relatively weak inhibition on the activities of CYP2B6, CYP2C9, and CYP3A4, with $\mathrm{IC}_{50}$ values of $669.91 \mu \mathrm{g} / \mathrm{mL}, 451.07$ $\mu \mathrm{g} / \mathrm{mL}$, and $235.48 \mu \mathrm{g} / \mathrm{mL}$, respectively (Fig. 3 and Table 5).

\section{Effects of HHT on the UGTs activities}

In vitro luminescence-based UGT assays were performed to evaluate whether HHT influenced the activities of human UGT1A1, UGT1A4, and UGT2B7. Diclofenac, which was used as a positive control, inhibited the activities of UGT1A1 and UGT2B7 in a dose-dependent manner, with $\mathrm{IC}_{50}$ values of $169.96 \mu \mathrm{M}$ and $55.42 \mu \mathrm{M}$, respectively (Fig. 4 and Table 6). Lopinavir, which was used as a positive control, inhibited UGT2B7 in a dose-dependent manner, with an $\mathrm{IC}_{50}$ value of 46.09 $\mu \mathrm{M}$ (Fig. 4 and Table 6).

HHT dose-dependently inhibited the activity of UGT1A1, with an $\mathrm{IC}_{50}$ value of $232.30 \mu \mathrm{g} / \mathrm{mL}$ (Fig. 4 and Table 6). The activities of UGT1A4 and UGT2B7 were inhibited by HHT; however, inhibition at $1000 \mu \mathrm{g} / \mathrm{mL}$ did not reach $50 \%$ (Fig. 4 and Table 6).

\section{Discussion}

Owing to the increasing consumption of herbal medicines worldwide, the safety analysis of these medicines has been highlighted. Toxicological assessment is required to identify the adverse effects of herbal medicines and determine the limit of exposure at which such effects occur. In the present study, we evaluated the safety of HHT by in vivo sub-acute toxicological test and in vitro drug -metabolizing enzymes assay.

When male and female rats were orally administered HHT once a day at doses of 0,500 , 1000 , and $2000 \mathrm{mg} / \mathrm{kg}$ for 4 weeks, mortality and
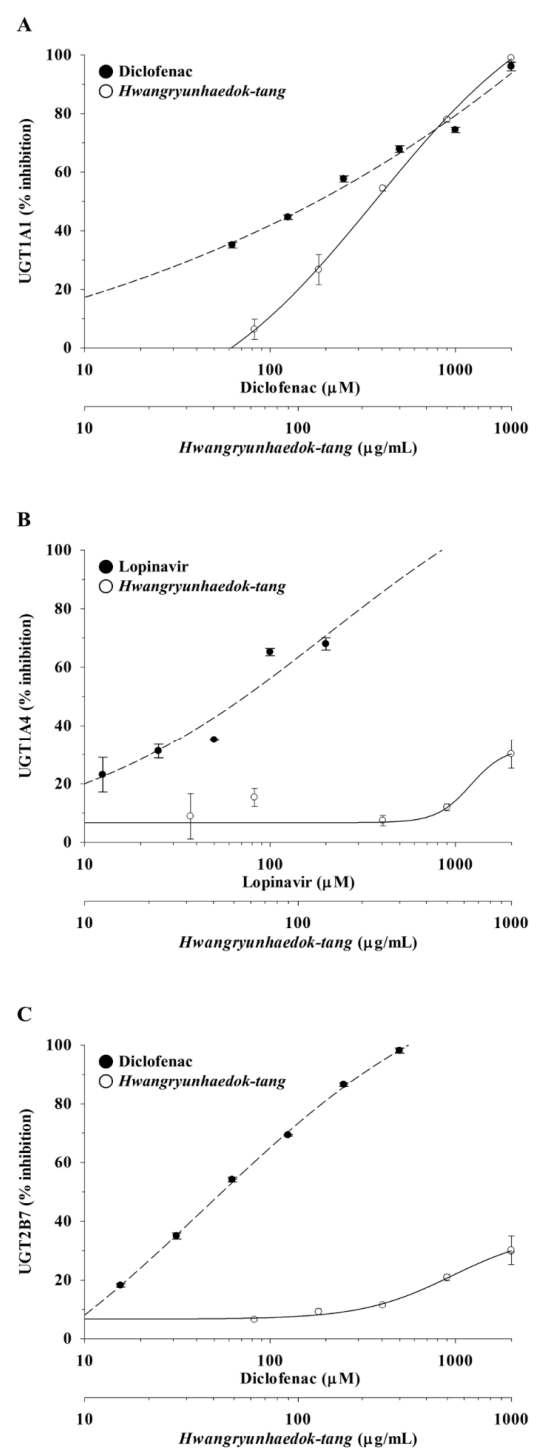

Fig. 4. The effects of Hwangryunhaedok-tang on the activities of UGT1A1 (A), UGT1A4 (B), and UGT2B7 (C).

Luminescence-based enzyme assays of human microsomal UGT isozymes were established in vitro. Diclofenac was used as a positive control for UGT1A1 and UGT2B7. Lopinavir was used as a positive control for UGT1A4. The data are presented as the means \pm SEM ( $n$ $=2$ ).

changes in body weight, food consumption, organ weights, hematology, and biochemistry were not observed. However, salivation in the 1000 and 2000 
Table 6. IC 50 values ( $\mu \mathrm{g} / \mathrm{mL})$ of Hwangryunhaedok-tang on UGT isozyme activities

\begin{tabular}{cccc}
\hline Herbal formula & UGT1A1 & UGT1A4 & UGT2B7 \\
\hline \hline Hwangryunhaedok-tang & 232.30 & $>1000$ & $>1000$ \\
Positive control & $169.96 \mu \mathrm{M}$ & $46.09 \mu \mathrm{M}$ & $55.42 \mu \mathrm{M}$ \\
\hline
\end{tabular}

Diclofenac was used as a positive control for UGT1A1 and UGT2B7. Lopinavir was used as a positive control for UGT1A4. The values are presented as the means of duplicate experiments.

$\mathrm{mg} / \mathrm{kg} /$ day male groups and $2000 \mathrm{mg} / \mathrm{kg} /$ day female group as well as loss of fur were observed; these symptoms are considered natural or accidental changes. The increase of bilirubin $(+1 \sim+2)$ and incidence of green urine in the $2000 \mathrm{mg} / \mathrm{kg}$ /day male and female groups are considered changes due to the administration of HHT. However, bilirubin levels $+1 \sim+2$ are detectable in normal animals, and there was no change in the biochemical and histopathological examination related to the kidney morphology or function. In addition, green urine is reported to occur during the elimination of administered materials such as drugs; it may also be caused by urinary tract infection of Pseudomonas aeruginosa $^{13,14)}$. In the present study, green urine was considered as a non-adverse effect because no symptoms associated with urinary tract infection were detected.

Significant differences were observed in the absolute weights of the kidneys, spleen, and ovaries of HHT-treated female group compared with those in the vehicle control group. However, these changes are not associated with HHT-induced abnormality because they are within normal ranges.

In the hematological test, the percentage of RET was significantly increased in the $2000 \mathrm{mg} / \mathrm{kg} /$ day female group, but not in the male groups. In addition, no change in other related hematological levels was observed. Therefore, the increase in percentage of RET is considered to be a non-adverse effect.

Although black discoloration of the kidneys and liver was considered the result of HHT treatment, there were no associated histopathological changes.
The black discoloration of the kidney is considered to be associated with green urine.

Histopathological examination was performed for organs with abnormal findings in necropsy, and no histopathological findings associated with HHT treatment were observed.

The key elements associated with successful drug development are efficacy and safety, and they are dependent on drug metabolism ${ }^{15}$. Changes in the activity or expression of drug-metabolizing enzymes can affect the concentration of a drug in the blood as well as its pharmacokinetic and medicinal properties ${ }^{16,17)}$. According to the report of Bush et al., adverse effects caused by herb-drug interactions were observed in $40 \%$ of patients receiving conventional therapy with an herbal product ${ }^{18)}$. Therefore, it is important to study the modulation of drug metabolizing enzymes by herbal formulas to understand the mechanism underlying herb-drug interactions.

Several reports have demonstrated the effects of HHT, the medicinal herbs or components present in HHT on the activities or levels of drug-metabolizing enzymes. HHT has been reported to inhibit human CYP1A2, CYP2C19, CYP2D6, and CYP3A4 ${ }^{19}$. Among the medicinal herbs present in HHT, Scutellariae Radix has been reported to inhibit human UGT1A1 activity ${ }^{20)}$. In addition, Scutellariae Radix increase and suppresses the levels of CYP1A and CYP2B in rats, respectively ${ }^{21)}$. In our previous study, chromatographic analysis of the five marker components, geniposide, baicalin, coptisine, palmatine, and berberine, in HHT was performed using an HPLC-PDA ${ }^{11)}$. Among the marker compounds, 
geniposide has been reported to suppress the mRNA and protein expression of CYP2E1 in mice ${ }^{22)}$, and inhibit the human CYP1A2 activity ${ }^{19}$. Berberine decreases the activities of CYP2C9, CYP2D6, and CYP3A4 in humans ${ }^{19,23)}$. It is reported that CYP1A2 and CYP2D6 may play a role in the metabolism of palmatine ${ }^{24)}$. However, the effects of HHT on the activities of UGTs have not been elucidated to date.

In the present study, the effects of HHT on the activities of human CYP450s (CYP1A2, CYP2B6, CYP2C9, CYP2C19, CYP2D6, CYP2E1, and CYP3A4) and UGTs (UGT1A1, UGT1A4, and UGT2B7) were examined to assess herb-drug interactions related to the use of HHT in clinic. HHT most potently inhibited the activity of CYP2D6, followed by the activities of CYP2C19, CYP2E1, and CYP1A2. These results are consistent with the results reported by Lee et al. ${ }^{19)}$. Furthermore, HHT moderately inhibited the CYP3A4 activity, while CYP2B6, CYP2C9, UGT1A1, UGT1A4, and UGT2B7-mediated metabolism was not affected. Therefore, care should be taken when HHT is co-administered with any inhibitor or substrate of CYP2D6. In addition, HHT may influence the metabolic reactions mediated by CYP2C19, CYP2E1, and CYP1A2. Attention should be paid when high-dose HHT is simultaneously taken with drugs that are metabolized by CYP3A4.

Ibuprofen and naproxen, which are non-steroidal anti-inflammatory drugs (NSAIDs), are metabolized by $\mathrm{CYP} 2 \mathrm{C}^{25}$. Therefore, HHT is unlikely to cause clinically relevant herb-drug interactions when co-administered with ibuprofen or naproxen for inflammatory diseases. Among the drugs used for the treatment of hypertension, nifedipine and simvastatin are substrates of CYP3A4, and tolbutamide and warfarin are metabolized by $\mathrm{CYP} 2 \mathrm{C} 9^{25,26)}$. In patients taking HHT for hypertension, the co-administration of tolbutamide or warfarin instead of nifedipine or simvastatin can reduce the possibility of herb-drug interactions. Cimetidine and omeprazole are widely used to treat gastric diseases.
Cimetidine is known to be an inhibitor of CYP1A2, CYP2C19, and CYP3A4, and omeprazole acts as a substrate of CYP2C19 and an inhibitor of CYP2C19 and CYP3A4. Ticlopidine, which is used to treat cerebrovascular diseases, has been reported to inhibit CYP1A2, CYP2C9, CYP2C19, and CYP2D6 ${ }^{25}$. Therefore, to reduce any adverse effects, caution should be exercised when HHT is co-administered with cimetidine, omeprazole, or ticlopidine.

\section{Conclusions}

We previously reported the safety of HHT in a 13-week repeated-dose oral toxicity study in rats; however, the side effects of HHT have not been reported. In the present study, the NOAEL value of HHT administration for 4 weeks is determined as $2000 \mathrm{mg} / \mathrm{kg} /$ day in both sexes. In addition, HHT acts as an inhibitor of CYP1A2, CYP2C19, CYP2D6, and CYP2E1. Therefore, to reduce its adverse effects, caution should be exercised when HHT is co-administered with substrates or other inhibitors of CYP1A2, CYP2C19, CYP2D6 or CYP2E1. However, HHT has a relatively low potential to be involved in herb-drug interactions when taken along with substrates or inhibitors of CYP2B6, CYP2C9, CYP3A4, UGT1A1, UGT1A4, and UGT2B7.

\section{Acknowledgements}

This research was supported by the grants 'Construction of Scientific Evidences for Herbal Medicine Formulas (K17251)' from the Korea Institute of Oriental Medicine (KIOM).

\section{Competing interests}

The authors declare that they have no competing interests. 


\section{References}

1. Firenzuoli F, Gori L. Herbal medicine today: clinical and research issues. Evid Based Complement Alternat Med. 2007;4(Suppl 1):37-40.

2. Moreira DL, Teixeira SS, Monteiro MHD, De-Oliveira ACAX, Paumgartten FJR. Traditional use and safety of herbal medicines. Revista Brasileira de Farmacognosia. 2014;24(2):248-57.

3. Nebert DW, Russell DW. Clinical importance of the cytochromes P450. Lancet. 2002;360(9340): 1155-62.

4. Wienkers LC, Heath TG. Predicting in vivo drug interactions from in vitro drug discovery data. Nat Rev Drug Discov. 2005;4(10):825-33.

5. Court MH, Hazarika S, Krishnaswamy S, Finel M, Williams JA. Novel polymorphic human UDP-glucuronosyltransferase 2A3: cloning, functional characterization of enzyme variants, comparative tissue expression, and gene induction. Mol Pharmacol. 2008;74(3):744-54.

6. Mackenzie PI, Bock KW, Burchell B, Guillemette C, Ikushiro S, Iyanagi T, et al. Nomenclature update for the mammalian UDP glycosyltransferase (UGT) gene superfamily. Pharmacogenet Genomics. 2005;15(10):677-85.

7. Hur J. Donguibogam. Seoul:Namsandang. 2007: 382.

8. Lu J, Wang JS, Kong LY. Anti-inflammatory effects of Huang-Lian-Jie-Du decoction, its two fractions and four typical compounds. $\mathrm{J}$ Ethnopharmacol. 2011;134(3):911-8.

9. Ohta $\mathrm{Y}$, Kongo-Nishimura M, Hayashi $\mathrm{T}$, Kishikawa T. Effect of Oren-gedoku-to (Huanglian -Jie-Du-Tang) extract on disruption of hepatic antioxidant defense systems in rats treated with D-galactosamine. J Ethnopharmacol. 2004;94(2-3): 323-9.

10. Lee MY, Seo CS, Kim YB, Shin IS, Shin HK. Non-clinical safety assessment of Hwangryunhaedok -tang: 13-week toxicity in Crl:CD Sprague
Dawley rats. Regul Toxicol Pharmacol. 2014; 68(3):378-86.

11. Seo CS, Kim OS, Kim JH, Shin HK. Simultaneous quantification and antiatherosclerosis effect of the traditional Korean medicine, Hwangryunhaedok-tang. BMC Complement Altern Med. 2015;15:108.

12. Jin SE, Seo CS, Shin HK, Ha H. Traditional Herbal Formulas to as Treatments for Musculoskeletal Disorders: Their Inhibitory Effects on the Activities of Human Microsomal Cytochrome P450s and UDP-glucuronosyltransferases. Pharmacogn Mag. 2016;12(48):241-52.

13. Raymond JR, Yarger WE. Abnormal urine color: differential diagnosis. South Med J. 1988; 81(7):837-41.

14. Lee YC, Lee JN, Bae JS, Park YC. Green urine in a patient who received a continuous infusion of propofol. Kor J Anesthesiol. 2009;56:325-7.

15. Gonzalez Frank J, Tukey Robert H. Chapter 3, Drug metabolism. In: Brunton LL, Lazo JS, Parker KL. Goodman \& Gilman's the pharmacological basis of therapeutics, 11th ed. New York: McGraw Hill. 2006:71-91.

16. Wang BL, Li Y. Progress of pharmacokinetic interactions and possible mechanisms of botanical medicines-chemical drugs. Chin J Pharmacol Toxicol. 2008;22:237-40.

17. Flockhart DA, Oesterheld JR. Cytochrome P450-mediated drug interactions. Child Adolesc Psychiatr Clin N Am. 2000;9(1):43-76.

18. Bush TM, Rayburn KS, Holloway SW, Sanchez-Yamamoto DS, Allen BL, Lam T, et al. Adverse interactions between herbal and dietary substances and prescription medications: a clinical survey. Altern Ther Health Med. 2007;13(2):30-5.

19. Lee SY, Jang H, Lee JY, Ma JY, Oh SJ, Kim SK. Inhibitory effects of Hwang-Ryun-Hae-Dok -Tang on cytochrome P450 in human liver microsomes. Xenobiotica. 2015;45(2):131-8. 
20. Katoh M, Yoshioka Y, Nakagawa N, Yokoi T. Effects of Japanese herbal medicine, Kampo, on human UGT1A1 activity. Drug Metab Pharmacokinet. 2009;24(3):226-34.

21. Kang JJ, Chen YC, Kuo WC, Chen T, Cheng YW, Kuo ML, et al. Modulation of microsomal cytochrome $\mathrm{P} 450$ by Scutellariae Radix and Gentianae scabrae Radix in rat liver. Am J Chin Med. 1996;24(1):19-29.

22. Ma $\mathrm{T}$, Huang $\mathrm{C}$, Zong $\mathrm{G}$, Zha $\mathrm{D}$, Meng $\mathrm{X}$, Li $\mathrm{J}$, et al. Hepatoprotective effects of geniposide in a rat model of nonalcoholic steatohepatitis. J Pharm Pharmacol. 2011;63(4):587-93.

23. Guo Y, Chen Y, Tan ZR, Klaassen CD, Zhou
HH. Repeated administration of berberine inhibits cytochromes $\mathrm{P} 450$ in humans. Eur $\mathrm{J}$ Clin Pharmacol. 2012;68(2):213-7.

24. Vrba J, Papouskova B, Pyszkova M, Zatloukalova M, Lemr K, Ulrichova J, et al. Metabolism of palmatine by human hepatocytes and recombinant cytochromes P450. J Pharm Biomed Anal. 2015;102:193-8.

25. Ogu CC, Maxa JL. Drug interactions due to cytochrome P450. Proc (Bayl Univ Med Cent). 2000;13(4):421-3.

26. Marino MR, Vachharajani NN. Drug interactions with irbesartan. Clin Pharmacokinet. 2001;40(8): 605-14. 